\title{
Influence of positive ions on the current-voltage characteristics of MOS structures
}

\author{
A. G. Tangena, J. Middelhoek, and N. F. de Rooij \\ Solid State Electronics Laboratory, Twente University of Technology, P.O. Box 217, Enschede, \\ The Netherlands \\ (Received 10 November 1977; accepted for publication 21 December 1977)
}

\begin{abstract}
A new mathematical approach for the influence of mobile positive ions on the current-voltage characteristics of MOS structures is presented. This new method gives formulations which are more applicable than those described in the literature. Examples of the application of these formulations are presented.
\end{abstract}

PACS numbers: $73.40 . Q v, 77.50 .+p$

\section{INTRODUCTION}

Positive mobile ions in $\mathrm{SiO}_{2}$ layers cause instabilities in MOS devices. Therefore, the behavior of these ions has led to extensive studies. In a review paper, Deal ${ }^{1}$ gives the results of many investigations. For a proper interpretation of the results of experiments, it is necessary to know the equilibrium distribution of ions in the dielectric. A model for the charge distribution under conditions of equilibrium was described by Chou. ${ }^{2}$ He assumed that in the oxide of the MOS structure there are positive mobile ions as well as immobile negative ions. Marciniak and Przewlocki elaborated the model where there are only positive ions in the oxide. ${ }^{3-5}$ This situation corresponds with the experimental results reported in the literature. ${ }^{1,6}$ The mathematical approach of Chou, Marciniak, and Przewlocki is rather complex. In this paper another approach is presented which leads to formulations that are more easy to apply.

\section{FORMULATION OF THE PROBLEM}

The equilibrium distribution of space charge in the dielectric layer of an MOS structure (Fig. 1) is considered. The following is assumed:

(1) The dielectric layer of the MOS structure is uniform, and its thickness is small in comparison to the electrode diameter, so that the problem may be considered one dimensional.

(2) There is no charge exchange between the dielectric and the electrodes (ideal blocking electrodes).

(3) An uncompensated charge of positive mobile ions exists in the dielectric. There is no charge generation and recombination in the dielectric layer. The calculation is based on the fact that the charge distribution, even when it changes under the influence of an applied field, can be described by an equilibrium distribution. This implies that the electrochemical potential $\tilde{\mu}_{i o n}$ of an ion everywhere in the oxide has the same value. Thus,

$$
\tilde{\mu}_{\text {ion }}=E_{o}+q V(x)+k T \ln \left[N(x) / N_{o}\right]=\text { const. }
$$

In Eq. (1), $E_{o}$ denotes the energy level of positive ions in the oxide, $q$ is the electron charge, $V(x)$ is the electrical potential at position $x, k$ is Boltzmann's constant, $T$ is the absolute temperature, $N(x)$ is the ion concentration at position $x$, and $N_{0}$ is the state density available for the ion.
Equation (1) corresponds with the assumption that the ions are distributed according to Boltzmann's equation. The number of occupied states is small compared to those available. Thus, $N(x)$ is much smaller than $N_{0}$, and we are allowed to use the theory for dilute solutions.

If we differentiate Eq. (1), we obtain

$$
\frac{d \tilde{\mu}_{\text {ion }}}{d x}=0=q \frac{d V(x)}{d x}+\frac{k T}{N(x)} \frac{d N(x)}{d x},
$$

and if we substitute the electric field $E(x)$ for the negative gradient of the potential, Eq. (2) becomes

$$
\frac{k T}{q} \frac{d N(x)}{d x}-E(x) N(x)=0 .
$$

In combination with Poisson's equation,

$$
\frac{d E(x)}{d x}=\frac{q}{\epsilon} N(x)
$$

( $\epsilon$ denotes the permitivity of the dielectric) we can calculate the equilibrium charge distribution.

Chou, Marciniak, and Przewlocki combined Eqs. (3) and (4) so that they obtained a differential equation for

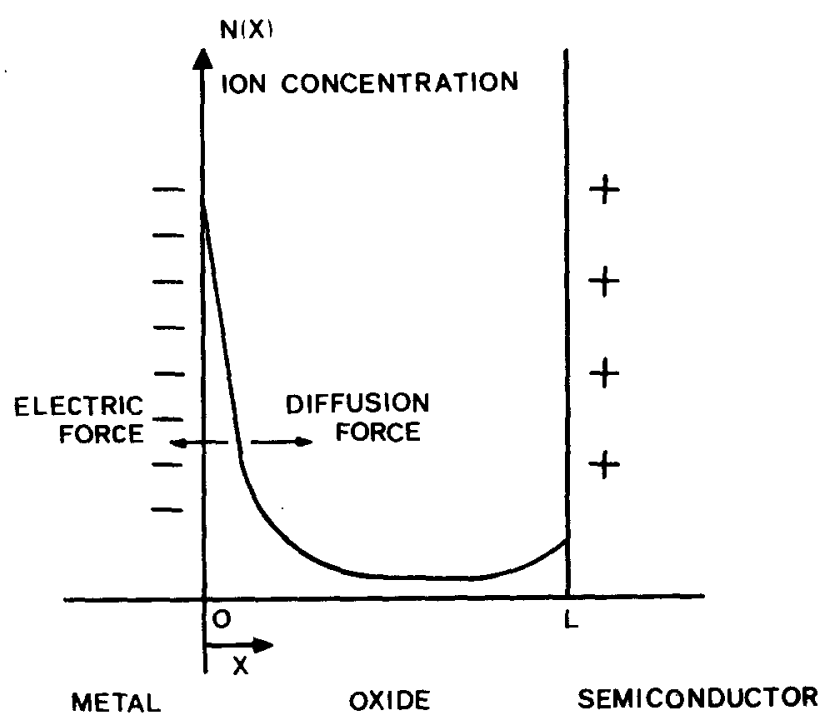

FIG. 1. Schematic representation of the MOS structure described. 


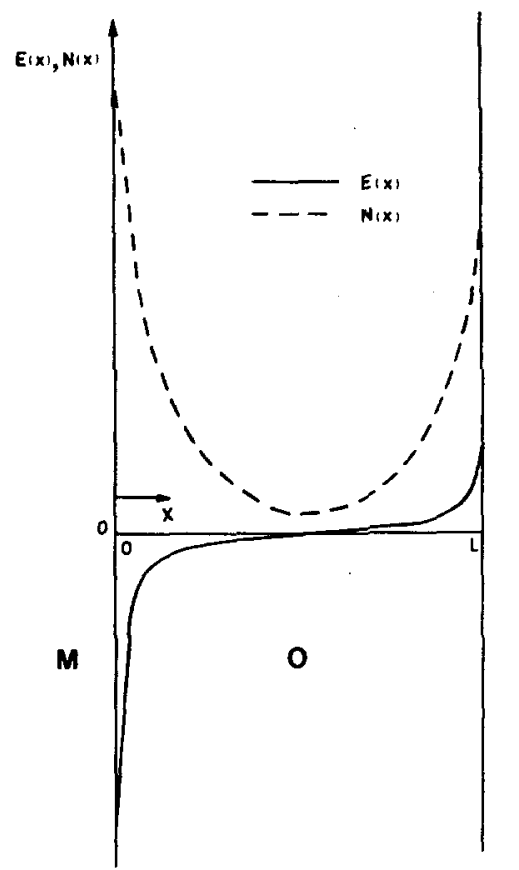

FIG. 2. The electric field $E(x)$ and the ion distribution $N(x)$ in the oxide of the MOS structure.

the concentration $N(x)$. This equation proved very difficult to solve. In this paper, Eqs. (3) and (4) are combined to give a differential equation in the electric field $E(x)$. This last differential equation is easy to solve. Once the electric field is calculated, Poisson's equation can be used to obtain the ion concentration. Elimination of $N(x)$ in Eq. (3) with the aid of Eq. (4) gives

$$
\frac{k T}{q} \frac{d^{2} E(x)}{d x^{2}}-E(x) \frac{d E(x)}{d x}=0 .
$$

If we substitute

$$
E(x) \frac{d E(x)}{d x}=\frac{1}{2} \frac{d E(x)^{2}}{d x},
$$

Eq. (5) can be integrated to give

$$
\frac{2 k T}{q} \frac{d E(x)}{d x}-E(x)^{2}=C_{1},
$$

where $C_{1}$ is an integration constant. Conversion of Eq. (7) leads to

$$
\frac{2 k T}{q} \int_{E} \frac{d E(x)}{E(x)^{2}+C_{1}}=\int_{0}^{x} d x=x .
$$

The integral in Eq. (8) is a standard integral with three different solutions, depending on the value of the integration constant $C_{1}$. For $C_{1}>0, \mathrm{Eq} .(8)$ gives

$$
\frac{2 k T}{q}\left[\frac{1}{\left(C_{1}\right)^{1 / 2}} \arctan \left(\frac{E(x)}{\left(C_{1}\right)^{1 / 2}}\right)\right]+C_{2}=x ;
$$

for $C_{1}=0$, Eq. (8) gives

$$
\frac{2 k T}{q}\left(\frac{-1}{E(x)}\right)+C_{3}=x ;
$$

for $C_{1}<0$, Eq. (8) gives

$$
\frac{2 k T}{q}\left[\frac{1}{2\left(-C_{1}\right)^{1 / 2}} \ln \left(\frac{E(x)-\left(-C_{1}\right)^{1 / 2}}{E(x)+\left(-C_{1}\right)^{1 / 2}}\right)\right]+C_{4}=x,
$$

where $C_{2}, C_{3}$, and $C_{4}$ are integration constants.

First the electric field $E(x)$ is calculated using Eq. (9),

$$
E(x)=(2 a k T / q) \tan (a x+b),
$$

where

$$
a=\frac{q\left(C_{1}\right)^{1 / 2}}{2 k T}
$$

and

$$
b=\frac{q\left(C_{1}\right)^{1 / 2}}{2 k T} C_{2}
$$

Now, it is simple to obtain the ion concentration $N(x)$ with Poisson's equation (4),

$$
N(x)=\frac{2 k T \epsilon}{q^{2}} \frac{a^{2}}{\cos ^{2}(a x+b)} .
$$

Graphic solutions of Eq. (12) and (15) are represented in Fig. 2. With Eq. (15), the major part of the possible ion distributions can be described. The solutions with $C_{1}=0$ or $C_{1}<0$ correspond with situations where the ions are located only at one of the interfaces (see Fig. 3 ). Solution (9) with $C_{1}>0$ is most important for the calculation of transport properties of ions in the oxide. Therefore, our calculations are limited to the condition $C_{1}>0$. The value of the two constants $a$ and $b$ in Eqs. (13) and (14) can be determined with the aid of the following boundary conditions:

$$
\begin{aligned}
\int_{0}^{L} E(x) d x & =-V_{0} \\
& =\text { potential difference over the oxide. } \\
V_{0}= & \frac{-2 k T}{q} \ln \left(\frac{\cos b}{\cos (a \bar{L}+b)}\right) \\
= & \frac{-2 k T}{q} \ln \left(\frac{\cos b}{\cos a L \cos b-\sin a L \sin b}\right) \\
Q_{0}= & \epsilon \int_{E(0)}^{E(L)} d E \\
= & \frac{2 a \epsilon k T}{q}[\tan (a L+b)-\tan b]
\end{aligned}
$$

where $Q_{0}$ is the assumed total charge per unit area. The unknown constants $a$ and $b$ are given implicitly in Eqs. (16) and (17). A combination of Eqs. (16) and (17) with the use of some goniometric formulas leads to
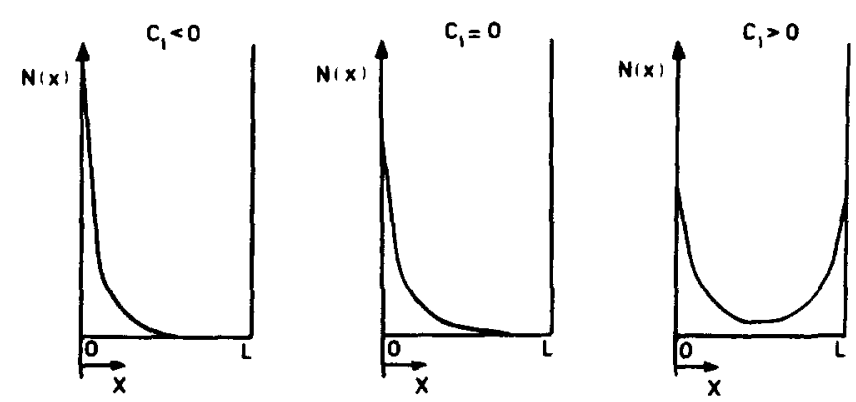

FIG. 3. Ion distribution $N(x)$ at different values of the integration constant $C_{1}$. 


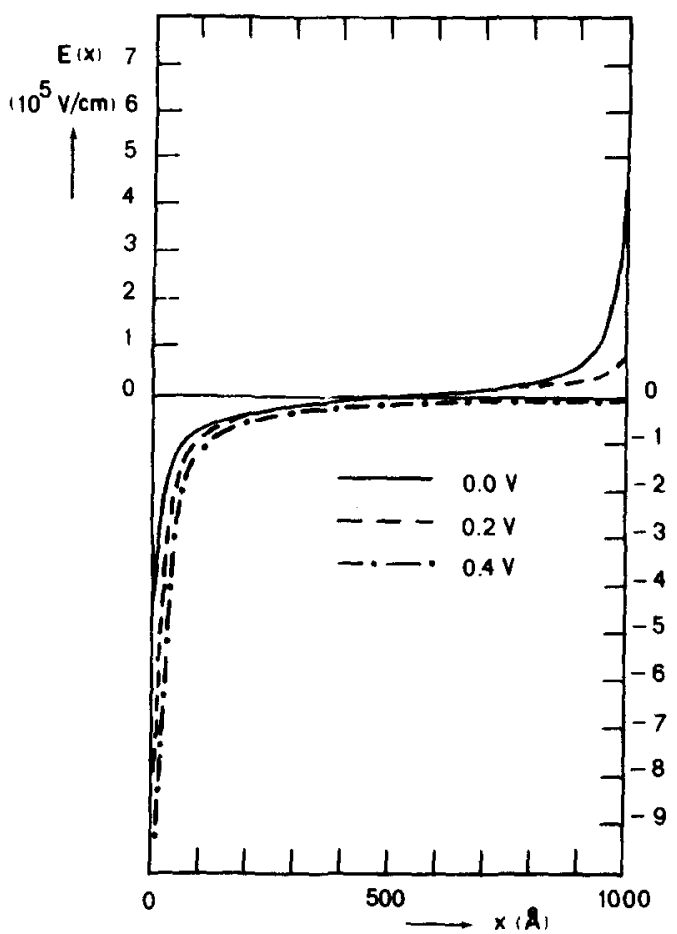

FIG. 4. The electric field $E(x)$ in the oxide at different applied voltages across the oxide $\left(T=250^{\circ} \mathrm{C} ; Q_{0} / q=2 \times 10^{12}\right.$ ions $\left./ \mathrm{cm}^{2}\right)$.

$$
\begin{aligned}
& \left(q Q_{0} L / 2 \epsilon k T\right) \exp \left(-\frac{1}{2} y\right) \sin (a L) \\
& \quad=a L\left[1+\exp (-y)-2 \exp \left(-\frac{1}{2} y\right) \cos (a L)\right],
\end{aligned}
$$

where $y=q V_{0} / k T$.

Once the constant $a$ is determined with an iterative process, we can use Eq. (16) to obtain the value of $b$. Equations (12) and (15) then give us the electric field and the ion concentration in the oxide (see Figs. 4 and $5)$.

\section{USE OF THE EQUILIBRIUM DISTRIBUTION $N(x)$ TO CALCULATE I-V CURVES}

The foregoing theory can be used to calculate the influence of the transport of ions in the oxide on the $I-V$ characteristics of a MOS structure. ${ }^{7}$ It is assumed that if the change of the applied voltage across the oxide is sufficiently slow (quasistatic approach), the real ion distribution can always be approximated by the equilibrium distribution. An $I-V$ curve is then calculated from a succession of equilibrium states. This calculation can be simplified by taking the capacitance of the space-charge layer of the semiconductor to be large compared to the oxide capacitance $C_{0 x}$. This is the case if the voltage drop in the semiconductor is negligible, for example, when the temperature is high or the semiconductor is heavily doped, or the surface is in accumulation. Referring to Fig. 1 , the charge $Q(L)$ at the semiconductor surface can be calculated

$$
\begin{aligned}
Q(L) & =C_{\mathrm{ox}} V_{0}+q \int_{0}^{L} \frac{x N(x)}{L} d x \\
& =C_{\mathrm{ox}} V_{0}+q \epsilon \int_{E(0)}^{E(L)} \frac{x}{L} d E(x),
\end{aligned}
$$

where $q[x N(x) / L]$ is the charge at position $x=L$, which

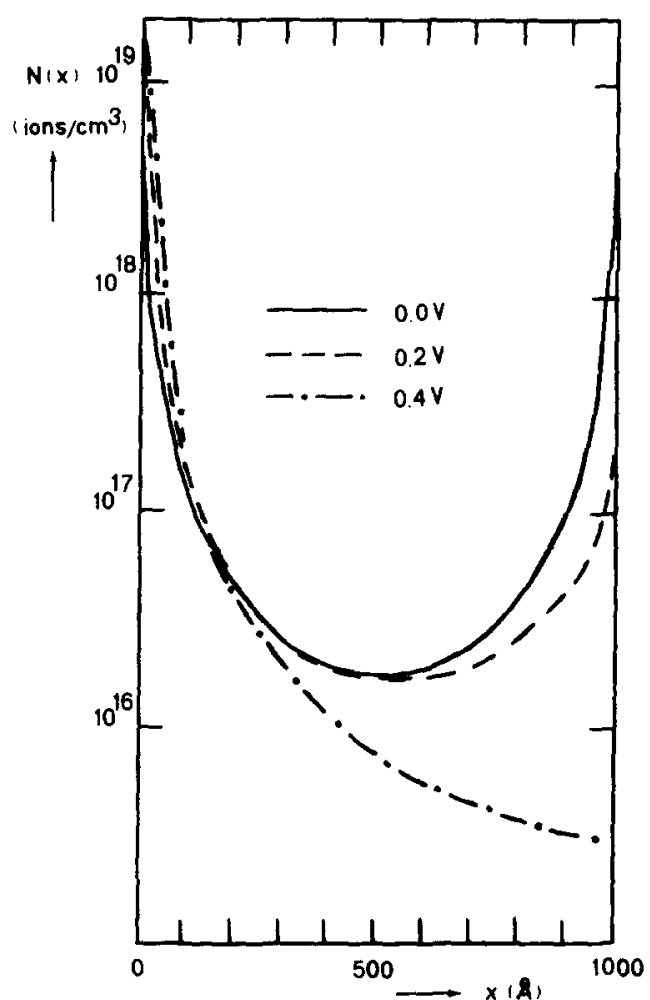

FIG. 5. The ion distribution $N(x)$ in the oxide at different applied voltages across the oxide $\left(T=250^{\circ} \mathrm{C} ; Q_{0} / q=2 \times 10^{12}\right.$ ions/ $\mathrm{cm}^{2}$ ).

is induced by ions at $x$ (negative image charge). If we substitute Eq. (12) into Eq. (19), we obtain

$$
\begin{aligned}
Q(L)= & C_{o x} V_{0}+\frac{2 k T \epsilon}{q L}[a L \tan (a L+b) \\
& \left.-\ln \left(\frac{1}{\cos (a L+b)}\right)+\ln \left(\frac{1}{\cos b}\right)\right] .
\end{aligned}
$$

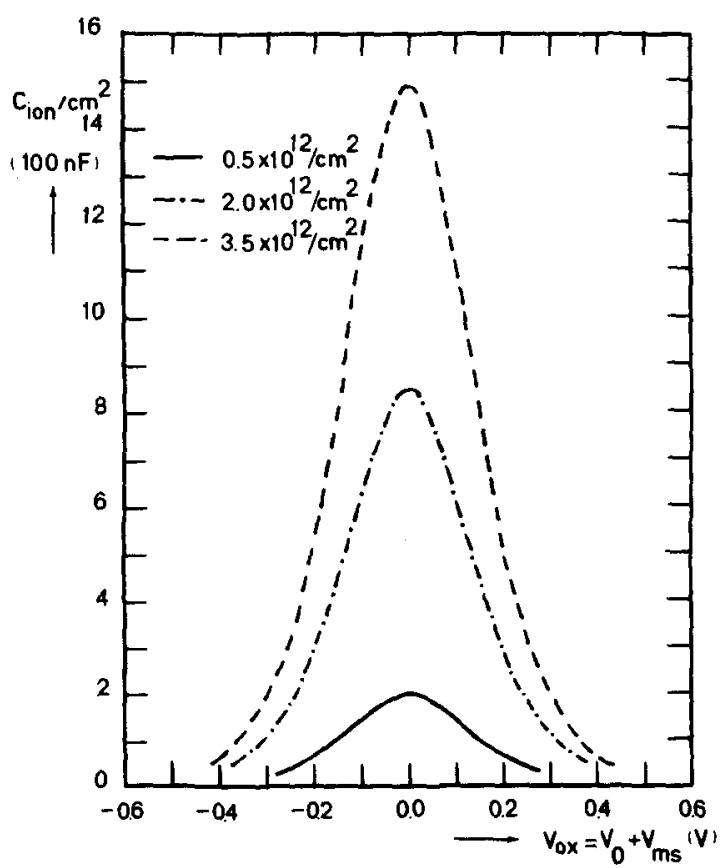

FIG. 6. The capacitance $C_{10 \text { caused by ion transport within }}$ the oxide at different total ion concentrations $\left(T=250^{\circ} \mathrm{C} ; L\right.$ $\left.=1000 \AA ; \epsilon_{o x}=3.9\right)$. 


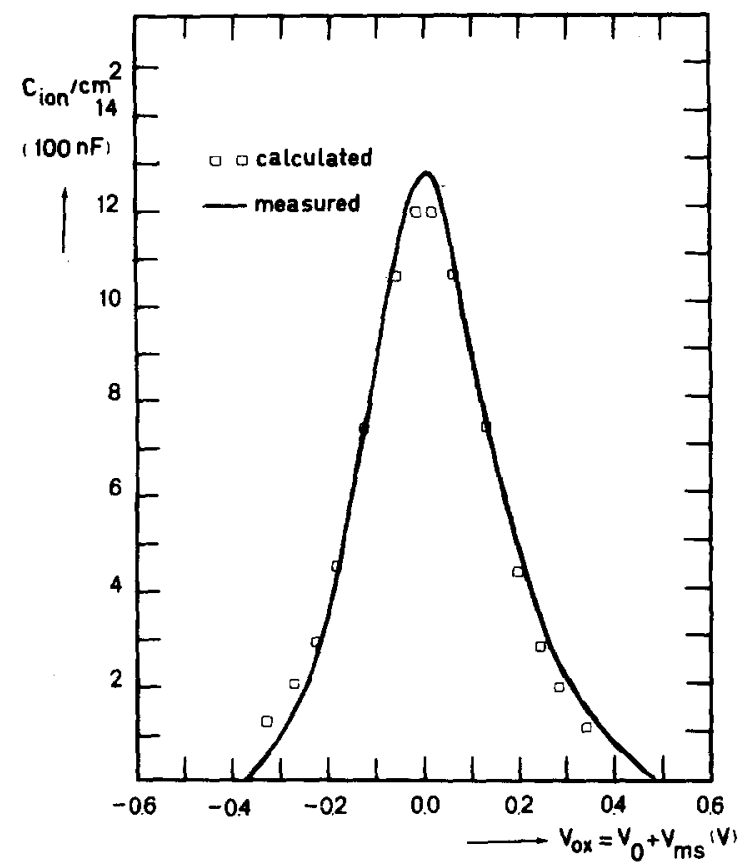

FIG. 7. A comparison of a calculated and a measured $C-V$ curve for an $\mathrm{Al} / \mathrm{SiO}_{2} / n^{++} \mathrm{Si}(100)$ structure. Contamination with $\mathrm{Na}^{+}$ions $\left(T=250^{\circ} \mathrm{C} ; L=1000 \AA ; \epsilon_{\alpha \alpha}=3.9\right.$; sweep rate: $30 \mathrm{mV} / \mathrm{s}$; $Q_{0} / q=3 \times 10^{12}$ ions $\left./ \mathrm{cm}^{2}\right)$.

With Eq. (20), the charge in the semiconductor at position $x=L$ can be calculated at a given voltage $V_{0}$ and a total charge per unit area $Q_{0}$. With the aid of numerical methods, it is now simple to calculate $\Delta Q(L) / \Delta V_{0}$. In this way, the $C-V$ curves can be calculated (Fig. 6 ) as well as the $I-V$ characteristics, when the voltage changes linearly with the time. Since the MOS behavior is symmetric around $V_{0 x}=0 \mathrm{~V}$, the cal- culation for one polarity of the voltage is sufficient. In practice, the situation $V_{0 x}=0 \mathrm{~V}$ is found at an applied external voltage $V_{\text {electroco }}$ equal to $V_{\text {ms }}$, the workfunction difference between electrode and semiconductor. Therefore, the top of the peak in the $I-V$ curve is situated not at $V_{\text {electrode }}=0 \mathrm{~V}$, but at $V_{\text {electrode }}=-V_{m s}$ (assuming there is no voltage drop in the semiconductor). In Fig. 7, we compare a measured $C-V$ curve with a calculated curve. For this calculation, $Q_{0}$ is determined by integration of the experimental curve. It is clear that with the above theory a reasonable fit between experimental and theoretical results can be obtained.

\section{CONCLUSIONS}

It has been shown that it is possible to calculate the equilibrium distribution of ions in a dielectric in a simpler way than those which have been published in literature. The model described here gives $C-V$ curves that closely resemble experimentally found $C-V$ curves.

\section{ACKNOWLEDGMENT}

This work was supported by the Netherlands Organization for the Advancement of Pure Research (ZWO).

${ }^{1}$ B. E. Deal, J. Electrochem. Soc. 121, 198C (1974).

${ }^{2}$ N.J. Chou, J. Electrochem. Soc. 118, 601 (1971).

${ }^{3}$ W. Marciniak and H.M. Przewlocki, Phys. Status Solidi A 24, 359 (1974).

${ }^{4}$ H. M. Przewlocki and W. Marciniak, Phys. Status Solidi A 29, 265 (1975).

${ }^{5}$ W. Marciniak and H.M. Przewlocki, J. Electrochem. Soc. 123, 1207 (1976)

${ }^{6}$ T.W. Hickmott, J. Appl. Phys, 46, 2583 (1975).

${ }^{7}$ M. Kuhn and D.J. Silversmith, J. Electrochem. Soc. 118, 966 (1971). 